\title{
Gynecological History in Chronic Fatigue Syndrome: A Population-Based Case-Control Study
}

\author{
Roumiana S. Boneva, M.D., Ph.D., Elizabeth M. Maloney, M.S., Dr.P.H., Jin-Mann Lin, Ph.D., \\ James F. Jones, M.D., ${ }^{1}$ Friedrich Wieser, M.D., ${ }^{2}$ Urs M. Nater, Ph.D., ${ }^{1,3}$ \\ Christine M. Heim, Ph.D.,", and William C. Reeves, M.D., M.Sc."
}

\begin{abstract}
Background: Chronic fatigue syndrome (CFS) affects disproportionately more women than men, and the condition is more common at perimenopause. We examined gynecological history events as risk factors for CFS. Methods: In a case-control study from a randomly selected population sample from Wichita, Kansas, 36 women with CFS and 48 nonfatigued controls, of similar age, race, and body mass index (BMI), answered a structured gynecological history questionnaire.

Results: CFS cases and controls had the same mean age (51 years) and age at menarche (12 years). Overall, a greater proportion of women with CFS than controls reported pelvic pain unrelated to menstruation $(22.2 \%$ vs. $1.7 \%, p=0.004)$, endometriosis $(36.1 \%$ vs. $16.7, \%, p=0.046)$, and periods of amenorrhea ( $53.9 \%$ vs. $46.2 \%$, $p=0.06)$. Compared to controls, women in the CFS group had a higher mean number of pregnancies (2.8 vs 2.0, $p=0.05)$ and gynecological surgeries (1.8 vs. 1.1, $p=0.05)$. Similar proportions of the CFS $(69.4 \%)$ and control (72.9\%) groups were menopausal. Although menopausal women in the CFS and control groups had similar mean age (55.5 and 55.8, respectively), menopause occurred about 4.4 years earlier in the CFS group (41.7 years vs. 46.1 years, respectively, $p=0.11$ ). Among menopausal women, $76 \%$ of the CFS group reported hysterectomy vs. $54.6 \%$ of controls $(p=0.09)$, and $56 \%$ of women with CFS reported oophorectomy vs. $34.3 \%$ of controls $(p=0.11)$.

Conclusions: The higher prevalence of gynecological conditions and gynecological surgeries in women with CFS highlights the importance of evaluating gynecological health in these patients and the need for more research to clarify the chronologic and the pathophysiological relationships between these conditions and CFS.
\end{abstract}

\section{Introduction}

C HRONIC FATIGUE SYNDROME (CFS) is a serious disabling illness, and about $70 \%-80 \%$ of people with CFS identified in both community-based and tertiary care-based case-control studies are women. ${ }^{1-3}$ The prevalence of CFS is highest in the $40-49$-year-old age range. ${ }^{3}$ About $5 \%$ of women between 40 and 60 years of age in metropolitan, urban, and rural populations in central Georgia describe symptoms of CFS, and almost $3 \%$ of women in this age range meet strict clinical criteria for the illness. ${ }^{4-7}$ In spite of the preponderance of CFS in women of perimenopausal and menopausal ages, there is a paucity of literature about associations of gynecological factors with CFS.
There have been just a few research studies supporting a higher frequency of gynecological conditions in CFS. One study conducted in 1994 found that women with CFS (identified between 1989 and 1993 from physician practices in Atlanta, Georgia) were significantly more likely to have had a hysterectomy than were randomly selected age/race-matched community controls. ${ }^{8}$ Another study, conducted between September 1992 and December 1993, attempted to enroll all women between the ages of 21 and 73 who attended a Boston fatigue clinic between July 1984 and December 1993. They were retrospectively diagnosed with CFS by criteria of the 1988 CFS definition ${ }^{9}$ and were invited to participate in a casecontrol study; $51 \%$ completed a questionnaire on reproductive health. Controls were obtained among female outpatients of

\footnotetext{
${ }^{1}$ Centers for Disease Control and Prevention, Atlanta, Georgia.

${ }^{2}$ Department of Obstetrics and Gynecology, Emory School of Medicine, Atlanta, Georgia.

${ }^{3}$ Department of Psychiatry, University of Zurich, Zurich, Switzerland.

${ }^{4}$ Department of Psychiatry, Emory University, Atlanta, Georgia.
} 
the Internal Medicine Department of the same hospital, and $66 \%$ completed the questionnaire. Women with CFS were more likely than controls to have reported a history of menstrual problems, galactorrhea, endometriosis, uterine fibroids, polycystic ovary syndrome, sexually transmitted diseases (STD) (including pelvic inflammatory disease [PID]), and cervical problems. ${ }^{9}$ The authors speculated that frequent anovulatory cycles associated with irregular menses and decreased progesterone, suggested by the high prevalence of polycystic ovary syndrome, contributed to an elevated estrogen/progesterone ratio among women with CFS, and that this caused chronic immune activation. ${ }^{9}$ There is a need to replicate these studies in a population-based case-control study design.

The present study sought to identify factors associated with CFS and generate a hypothesis by examining differences in gynecological history between women with CFS (cases) and well/nonfatigued controls randomly recruited from the population of Wichita, Kansas.

\section{Materials and Methods}

\section{Subjects}

This study adhered to U.S. Department of Health and Human Services (DHHS) human experimentation guidelines and received Institutional Review Board approval from the Centers for Disease Control and Prevention (CDC) and collaborating institutions. All participants gave informed consent. Data were collected during an in-hospital case-control study of persons with CFS and nonfatigued (NF) controls. In brief, the in-hospital study enrolled participants from the Wichita CFS Surveillance study conducted in 1997-2000. 7,10 The Surveillance study used a random digit-dialing telephone survey to screen 56,146 adult residents $18-69$ years of age in Wichita (Fig. 1). The telephone screening (in 1997) identified people who had fatigue for a month or more $(n=5295)$ and those who did not and was followed by a detailed telephone interview of those with fatigue $(n=3528$ participants) and of 3634 randomly selected people without fatigue. That cohort of 7162 individuals was contacted for annual interviews between 1998 and 2000 (Fig. 1). All who met CFS criteria on the phone (CFS-like) were invited for a clinical evaluation along with: (1) a similar number of randomly selected nonfatigued persons matched to the CFS-like on age, sex, race, and body mass index (BMI) and (2) a random sample of persons with fatigue of $\geq 6$ months but not meeting full CFS criteria (fatigued, unwell). The clinical evaluation included medical history, physical examination, laboratory tests, and psychiatric assessment and served to rule out exclusionary conditions and classify subjects as (1) CFS, (2) well, nonfatigued (NF) controls, and (3) persons with fatigue and some symptoms but not meeting full CFS criteria, named insufficient symptoms/fatigue (ISF).

In 2002, the study concluded with a 2-day in-clinic evaluation in a Wichita research hospital unit. Invited for participation were 70 people classified as having CFS at any point during the 4-year Surveillance study (58, 83\%, enrolled); 41 people with CFS and melancholic depression (27 enrolled); 70 NF controls matched to a person with CFS on sex, age, race, and BMI (55 enrolled); a random selection of 70 Surveillance participants who had unexplained fatigue for $\geq 6$ months during the 4-year surveillance but who did not meet full CFS criteria (ISF) in the past $(59,84 \%$, enrolled); and 39 people with
ISF and melancholic depression (28 enrolled), that is, a total of 227 participants. On admission to the hospital, subjects underwent reevaluation of current CFS symptoms and exclusionary conditions (for a detailed description of the study and specifics of CFS and NF classification, see reference 7). Figure 1 shows the final distribution of participants. Only the 36 women with CFS and the $48 \mathrm{NF}$ women (controls) are the subjects of comparison in this study.

\section{Classification standards for CFS and control (NF)}

Following exclusion of certain medical and psychiatric conditions, participants were classified as CFS or NF controls. We used criteria of the 1994 International CFS Research Case Definition, ${ }^{5}$ which were evaluated according to recommendations of the International CFS Study Group. ${ }^{6}$ Specifically, the study used internationally standardized, validated instruments to evaluate functional impairment (by the ShortForm Health Survey, [SF-36]), ${ }^{11}$ fatigue (by the Multidimensional Fatigue Inventory [MFI]) ${ }^{12}$ and frequency and severity of the eight CFS-defining symptoms (CDC Symptom Inventory). ${ }^{13}$ Classification as a current CFS case was based on cutoff scores derived from these rating scales with respect to the three-dimensional criteria of CFS specified in the case definition, that is, impairment, fatigue, and accompanying symptoms (for more details, see reference 7). Subjects meeting all three criteria at the time of the study were classified as CFS cases; those who met none of the criteria were classified as NF controls. ${ }^{7}$ Participants who met some but not all three criteria for CFS constituted the ISF, a separate group that is not included in the current analysis. Because invited controls were individually matched to cases with a CFS diagnosis during the past 4 years and subjects were reclassified according to current diagnostic status, strict matching could not be maintained. However, cases and controls were demographically similar.

\section{Data collection}

As part of the study's in-hospital clinical evaluation, during the first day on the ward, all women completed a selfadministered gynecological history questionnaire that queried age at menarche and menopause, duration of menstrual periods, bleeding between periods, cessation of periods for reason other than menopause, presence of lower abdominal/ pelvic pain unrelated to menstruation, number of pregnancies, menopause, use of hormone replacement (yes or no), history of gynecological conditions, and surgeries. There were two separate questions for hysterectomy and for removal of ovaries (oophorectomy). Subjects were asked if they had had "other gynecological surgeries," and if the answer was yes, the type of surgeries was queried. Questions about duration of menstrual period and bleeding between periods were answered only by premenopausal women. Physical examination in the clinic focused on identifying comorbidities exclusionary for CFS and did not include a pelvic examination. Review of medical records was not included in this study, as our major goals were to assess the prevalence of CFS in the community.

\section{Statistical methods}

Results are presented as mean and standard error of the mean (SEM) unless otherwise specified. We used the 


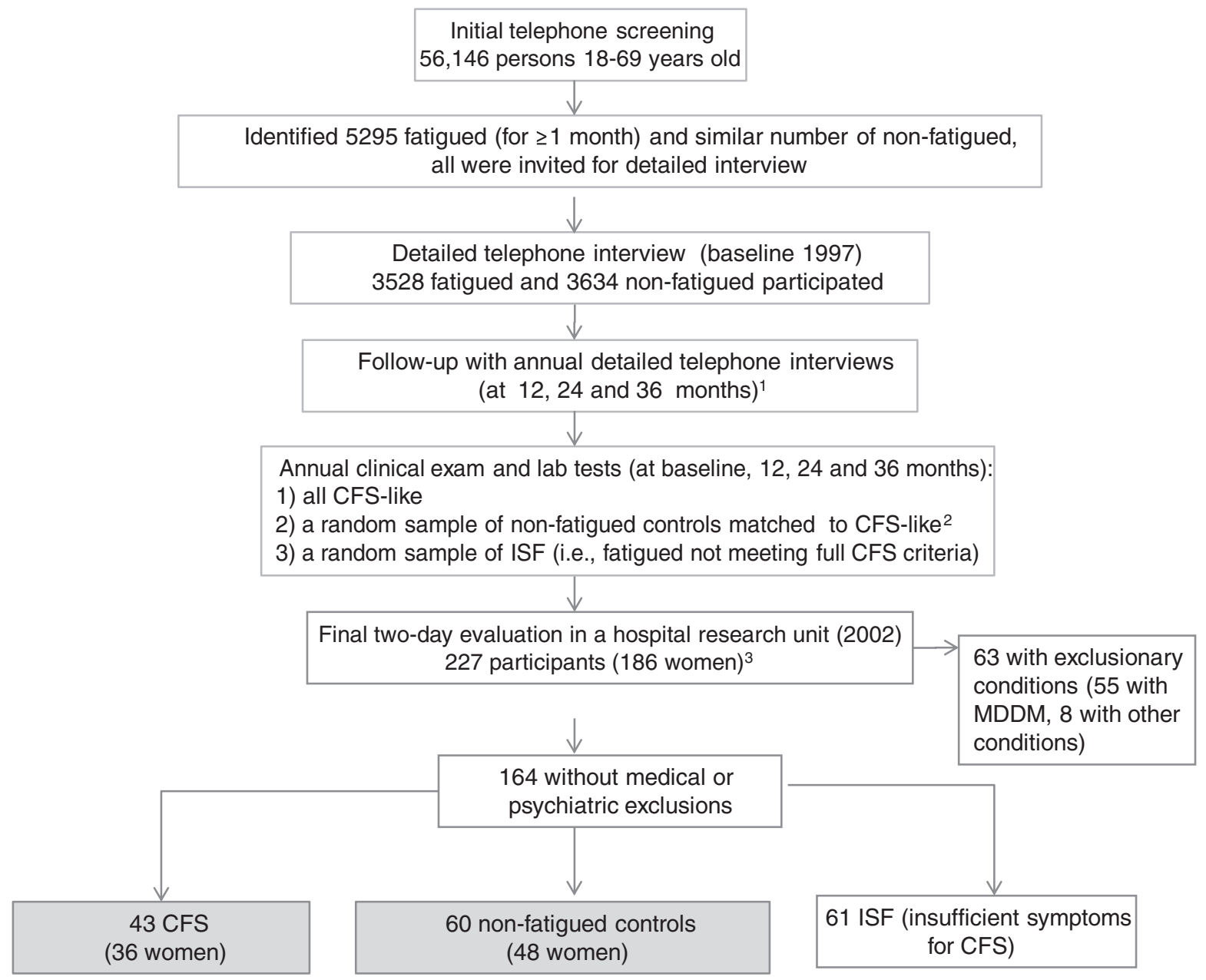

FIG. 1. Flow chart of the selection of participants in the Wichita Surveillance Study of Chronic Fatigue Syndrome, 19972002. ${ }^{1}$ The number of people who participated in the 12, 24, and 36 months follow-up (1998-2000) was 4228 in 1998,3980 in 1999 , and 3474 in $2000 .{ }^{2}$ Nonfatigued controls were matched to CFS on age, sex, race, and body mass index. ${ }^{3}$ See text for more details on the sample of 227 participants.

chi-square test to compare distribution of dichotomous variables between the CFS and the control groups. To compare continuous variables, which were not normally distributed, we used the nonparametric Wilcoxon test. We used logistic regression to assess the magnitude of the association of CFS with dichotomous variables. Exact methods were used to calculate the $95 \%$ confidence intervals (CIs) for odds ratios (ORs) when cell sizes were small. We calculated two-sided $p$ values to estimate the significance of associations. The statistical significance was set at alpha $=0.05$. However, we report all $p$ values and discuss all associations where the $p$ value was $<0.20$ because such associations may be worth examining in future studies, as this was an exploratory analysis for hypothesis generating and because the study had a relatively small sample size, limiting its power to detect small-size effects. We used SAS 9.1 (Cary, NC) statistical software.

\section{Results}

Women with CFS were demographically similar to the controls (Table 1). Comparisons of gynecological history variables between the CFS and control groups are presented in Table 2. More than two thirds of cases and controls were menopausal (Table 2). The CFS and control groups did not differ in mean age at menarche (12 years), bleeding between periods, or mean duration of menstrual periods.

Amenorrhea, pelvic pain unrelated to menstruation, endometriosis, any gynecological surgery, and specifically hysterectomy and oophorectomy, were all more common in the CFS than in the control group. These differences between

Table 1. Sample Characteristics of Women with Chronic Fatigue Syndrome and Controls, Wichita Case-Control Study of Chronic Fatigue Syndrome

\begin{tabular}{lccc}
\hline Variable & CFS $(\mathrm{n}=36)$ & Controls $(\mathrm{n}=48)$ & $\mathrm{p}$ \\
\hline Age $^{\mathrm{a}}$ & $50.9 \pm 1.5$ & $51.2 \pm 1.3$ & 0.86 \\
Race, $n(\%) n$ & & 0.21 \\
$\quad$ White & $33(91.7)$ & $47(97.9)$ & \\
$\quad$ Black & $1(2.8)$ & $1(2.1)$ & \\
$\quad$ Other & $2(5.5)$ & $0(0)$ & \\
Body mass index & & \\
& $29.5 \pm 0.7$ & $29.1 \pm 0.7$ & 0.68 \\
\hline
\end{tabular}

${ }^{\mathrm{a}}$ Mean \pm standard error of the mean (SEM).

CFS, chronic fatigue syndrome. 
Table 2. Comparison of Gynecological History of Women with Chronic Fatigue Syndrome AND Nonfatigued Controls

\begin{tabular}{|c|c|c|c|c|}
\hline Study group & $C F S(\mathrm{n}=36)$ & Controls $(\mathrm{n}=48)$ & OR $(95 \% C I)$ & $\mathrm{p}$ \\
\hline \multicolumn{5}{|l|}{ Gynecological variable } \\
\hline Age at menarche ${ }^{\mathrm{a}}$ & $12.3 \pm 0.2$ & $12.4 \pm 0.2$ & ND & 0.63 \\
\hline Duration of menstrual period $(n=23)^{\mathrm{b}}$ & $5.4 \pm 0.4$ & $5.0 \pm 0.5$ & ND & 0.55 \\
\hline Bleeding between periods ${ }^{c}$ & & & ND & 1.0 \\
\hline Yes & $1(9.1)$ & $1(7.7)$ & & \\
\hline No $(n=24)^{\mathrm{d}}$ & $10(90.9)$ & $12(92.3)$ & & \\
\hline \multicolumn{5}{|l|}{ Amenorrheac } \\
\hline Yes & $21(53.9)$ & $18(46.2)$ & $2.33(0.88-6.23)$ & 0.06 \\
\hline No & $15(46.1)$ & $30(53.8)$ & Reference & \\
\hline Number of pregnancies/woman ${ }^{a}$ & $2.8 \pm 0.3$ & $2.0 \pm 0.2$ & ND & 0.05 \\
\hline \multicolumn{5}{|l|}{ History of pelvic pain ${ }^{c}$} \\
\hline Yes & $8(22.2)$ & $1(1.7)$ & $13.43(1.61-608.2)$ & 0.004 \\
\hline No & $28(77.8)$ & $47(98.3)$ & Reference & \\
\hline \multicolumn{5}{|l|}{ History of endometriosis ${ }^{c}$} \\
\hline Yes & $13(36.1)$ & $8(16.7)$ & $2.83(1.02-7.83)$ & 0.046 \\
\hline No & $23(63.8)$ & $40(83.3)$ & reference & \\
\hline \multicolumn{5}{|l|}{ Other gynecological conditions ${ }^{c}$} \\
\hline Yes & $12(33.3)$ & $8(16.7)$ & $2.50(0.80-7.93)$ & 0.078 \\
\hline No & $24(66.7)$ & $40(83.3)$ & reference & \\
\hline \multicolumn{5}{|l|}{ Any gynecological surgery ${ }^{c}$} \\
\hline Yes & $28(77.8)$ & $26(54.2)$ & $2.96(1.02-8.79)$ & 0.045 \\
\hline No & $8(22.2)$ & $22(45.8)$ & Reference & \\
\hline Number of gynecological surgeries per person ${ }^{a}$ & $1.75 \pm 0.29$ & $1.08 \pm 0.20$ & ND & 0.05 \\
\hline \multicolumn{5}{|l|}{ History of other gynecological surgery ${ }^{c, e}$} \\
\hline Yes & $16(44.4)$ & $12(25.0)$ & $2.40(0.86-6.75)$ & 0.06 \\
\hline No & $20(55.6)$ & $36(75.0)$ & & \\
\hline Menopausal women $^{c}$ & $25(69.4)$ & $35(72.9)$ & & \\
\hline Mean age & $55.6 \pm 1.0$ & $54.9 \pm 1.1$ & ND & 0.65 \\
\hline Age at menopause (years) ${ }^{\mathrm{a}}$ & $41.7 \pm 2.3(n=20)$ & $46.1 \pm 1.5(n=28)$ & ND & 0.11 \\
\hline Age at natural menopause & $50.3 \pm 1.5(n=6)$ & $49.2 \pm 1.6(n=16)$ & ND & 0.68 \\
\hline Age at surgical menopause & $31.9 \pm 2.4(n=14)$ & $38 \pm 2.7(n=12)$ & ND & 0.29 \\
\hline Duration of reproductive period $^{\mathrm{a}}$ & $29.4 \pm 2.2(n=20)$ & $33.4 \pm 1.5(n=28)$ & ND & 0.13 \\
\hline \multicolumn{5}{|l|}{ Hysterectomy ${ }^{\mathrm{C}}$} \\
\hline Yes & $19(76)$ & $19(54.3)$ & $2.67(0.76-9.72)$ & 0.09 \\
\hline No & $6(24)$ & $16(45.7)$ & Reference & \\
\hline \multicolumn{5}{|l|}{ Oophorectomy $^{c}$} \\
\hline Yes & $14(56)$ & $12(34.3)$ & $2.44(0.75-8.06)$ & 0.11 \\
\hline No & $11(44)$ & $23(65.7)$ & Reference & \\
\hline
\end{tabular}

${ }^{\mathrm{a}}$ Mean \pm SEM.

b 11 women with CFS and 12 controls.

${ }^{c} n(\%)$.

${ }^{\mathrm{d}} 11$ women with CFS and 13 controls.

'e Includes dilation and curettage (D\&C), cesarean-sections, tubal ligations, and other.

$\mathrm{CI}$, confidence interval; $\mathrm{ND}$, net done; $\mathrm{OR}$, odds ratio.

CFS and controls were statistically significant for pelvic pain $(p=0.004)$, endometriosis $(p=0.046)$, and any gynecological surgery $(p=0.045)$ (Table 2$)$. In addition, the CFS group had a significantly greater mean number of pregnancies (2.8 in CFS vs. 2.0 in controls, $p=0.05)$ and greater mean number of gynecological surgeries (1.7 in CFS vs. 1.1 in controls, $p=0.05)$. Menopause occurred on average 4 years earlier in the CFS group ( $\sim 42$ years vs. 46 years in controls), but this difference was not statistically significant $(p=0.11)$ (Table 2 ). For the subset of women without a history of surgical menopause, however, the mean age at menopause was similar for the CFS and control groups (approximately 50 years) (table 2).

There was a strong association between endometriosis and pelvic pain (OR 15.3, 95\% CI 2.4-158.4, $p=0.0006$ ). In a mul- tiple logistic regression model, including both variables as CFS cofactors, pelvic pain remained strongly and significantly associated with CFS (OR 10.05, 95\% CI 1.1-91.73, $p=0.04$ ), whereas endometriosis did not remain statistically significantly associated with CFS (OR 1.7, 95\% CI 0.55-5.31, $p=0.36$ ); however, these calculations are based on a small number of control women with pelvic pain.

Women with CFS were significantly more likely than controls to report any gynecological surgery (OR 2.96, $p<0.05$ ). Overall, hysterectomy and oophorectomy were the most common surgeries. Among menopausal women with CFS, more than three quarters $(76.0 \%)$ reported hysterectomy, and more than half $(56.0 \%)$ reported oophorectomy. Menopausal women from the CFS group were more than twice as likely as menopausal controls to have had a hysterectomy (OR 2.67, 
$p=0.09$ ) or oophorectomy (OR 2.44, $p=0.11$ ) (Table 2), but the association was not statistically significant. Finally, reporting oophorectomy was strongly associated with reporting of endometriosis (OR 12.00, 95\% CI 3.24 - 44.42, $p=0.0002$ ), and this was equally true for the CFS group (OR 11.25, 95\% CI 1.65$76.84, p=0.014$ ) and for controls (OR 10.50, 95\% CI 1.69-66.09, $p=0.012$. In the overall sample of menopausal women $(n=60)$, the proportion of hormone replacement (HR) users in the CFS (64\%) and the control groups (57\%) did not differ significantly $(p=0.79)$. Among menopausal women who reported hysterectomy with oophorectomy, reported HR in the CFS group $(78.6 \%)$ was slightly lower compared with the control group $(83.3 \%)$ but not statistically different.

Apart from hysterectomy and oophorectomy, other gynecological surgeries were also more frequently reported by women with CFS, with a higher mean number of these surgeries in the CFS group ( $0.83 \pm 0.2$ vs. $0.42 \pm 0.13$ in controls, $p=0.10$ ). Of the other gynecological surgeries, dilation and curettage (D\&C) was the most common procedure $(19.4 \%$ of women with CFS and $4.2 \%$ of controls), followed by cesareansection and tubal ligation. The most common reason for D\&Cs in the CFS group was miscarriage: $11.1 \%$ of CFS subjects vs. $0 \%$ of controls (OR 2.50, 95\% CI undefined, $p=0.03$ by Fisher's exact test). Among other gynecological diseases or abnormalities (reported by $33 \%$ of CFS and $17 \%$ of controls, $p=0.078)$, pelvic inflammation/infection was the most common condition and was more frequently reported by the CFS group than by controls, but the difference was not statistically significant $(p=0.22)$.

\section{Discussion}

Our study is one of very few to examine the association between female gynecological history and CFS. We found a strong association between CFS and history of pelvic pain unrelated to menstrual periods. Pelvic pain is a major symptom of endometriosis, and this could explain its association with both endometriosis and CFS. When both were included in the model, however, only pelvic pain remained significantly associated with CFS. Another plausible explanation of this association could be that lower abdominal/pelvic pain is also present in other conditions comorbid with CFS, such as interstitial cystitis and irritable bowel syndrome (IBS). ${ }^{14,15}$

In our study, women with CFS were 2.8 times as likely as controls to report endometriosis, and the magnitude of the association was similar to that found in two previous studies. ${ }^{9,16}$ Endometriosis has been associated with CFS, autoimmune disorders, ${ }^{17}$ and such painful conditions as fibromyalgia (FM), IBS, and interstitial cystitis. ${ }^{14,15}$ It is, therefore, likely that CFS and these conditions may share common (yet not well understood) pathogenetic mechanisms. Most women with endometriosis in our study had undergone hysterectomy (with or without oophorectomy), and this made it practically impossible to characterize the relationship between CFS and endometriosis separately from hysterectomy/oophorectomy; in addition, dates of hysterectomy and oophorectomy were not available. In the endometriosis survey of Sinaii et al., ${ }^{17}$ the diagnosis of endometriosis preceded that of CFS, but there was no information about whether endometriosis had been surgically treated.

The association between CFS and a higher number of pregnancies is interesting, especially in view of the shorter mean reproductive period in the CFS group, but it is hard to interpret, as we did not know how many pregnancies ended in deliveries or miscarriages or when pregnancies occurred relative to CFS onset. The higher number of D\&Cs for miscarriage in the CFS group implies more fetal loss, but this speculation is based on a relatively small number of events. In a recent study, women with CFS had a lower occurrence of childbirths in the 3 years preceding their CFS diagnosis compared with controls (women with other diagnoses). ${ }^{18}$ If not due to avoidance of pregnancy, this finding suggests more fertility problems among women who later develop CFS or that pregnancy ending in childbirth may have a protective effect (possibly through the high levels of hormones of pregnancy). The association between CFS and number of pregnancies should also be reexamined in future studies. Our finding of a higher prevalence of amenorrhea unrelated to pregnancy in women with CFS is in agreement with the findings of Harlow et al. ${ }^{9}$ and may indicate the presence of ovarian hormone imbalances in women with CFS.

Women with CFS were almost three times as likely as controls to report gynecological surgeries (OR 2.96, $p<0.05)$, particularly hysterectomy (OR 2.67, $p=0.09$ ). Although not statistically significant, the association between hysterectomy and CFS in our study is in agreement with one previous casecontrol study from a physician-based CFS surveillance, ${ }^{8}$ which found a larger and statistically significant association between CFS and hysterectomy (OR 7.0, $p<0.05)$. Our results differ in that the magnitude of the association in that study by Reyes et al. ${ }^{8}$ was larger and the prevalence of hysterectomy, especially among controls, was lower (5\%) than in our study. Hysterectomy has been associated with higher BMI. ${ }^{19}$ In our study, controls were approximately matched to CFS cases on BMI; thus, our control group might have been skewed toward higher risk of hysterectomy, resulting in a potential underestimate of the association between CFS and hysterectomy.

It is striking that $76 \%$ of menopausal women with CFS in our study reported hysterectomy. This high prevalence of hysterectomies may explain, at least partially, the observed earlier onset of menopause in the CFS group. The hysterectomy rate among controls in our study $(39.6 \%)$ was comparable to the $34 \%-39 \%$ national and state data for similar age groups ${ }^{20-23}$ supporting the reliability of our data. Our study seems to be only the second to find an association between hysterectomy and CFS, whereas in FM, a condition that shares many symptoms with CFS, the association with hysterectomy has been better documented. ${ }^{24-27}$ For example, Wagener et al. ${ }^{25}$ showed that $53 \%$ of women with FM had hysterectomy compared with $28 \%$ of control women with other rheumatologic or internal medicine diagnoses and that hysterectomy preceded the diagnosis of FM by an average of 4 years in approximately three quarters of women. Similarly, ter Borg et al. ${ }^{26}$ found a significantly higher prevalence of hysterectomies that preceded newly diagnosed FM (32.8\% of cases) compared with controls with rheumatoid arthritis (14.6\%), even though the control group was older. Finally, Pamuk et al. ${ }^{27}$ reported a higher frequency of hysterectomy and of early (age $<45$ years) menopause in women with FM compared with women of the same age who had rheumatoid arthritis. Taken together, these findings suggest a pathogenetic mechanism that could be related to the hormonal changes occurring after hysterectomy/ oophorectomy or to the reason that led to hysterectomy. Women with CFS in our study were more than twice as likely 
as controls to have had oophorectomy. This association was not statistically significant $(p=0.11)$, but it deserves attention for hypothesis generation because bilateral oophorectomy (especially before onset of menopause) has been associated with various degenerative conditions, such as higher risk of cognitive impairment, ${ }^{28}$ neurological or mental diseases, ${ }^{29}$ unfavorable lipid profile, ${ }^{30}$ and cardiovascular disease. ${ }^{31,32}$ To elucidate the possible pathogenetic link between hysterectomy/oophorectomy and CFS, future larger studies should examine their chronological relationship.

Our findings and those reported in the literature associate CFS with higher prevalence of pelvic pain, amenorrhea, endometriosis, and gynecological surgeries. The mechanisms involved, as suggested in previous reports, may be estrogen/progesterone imbalance ${ }^{9}$ or deficiency. ${ }^{33}$ Harlow et al. ${ }^{9}$ speculated that frequent anovulatory cycles, associated with irregular menses and decreased progesterone (as suggested by the high prevalence of polycystic ovary syndrome in their study), contributed to a state of elevated estrogen/progesterone ratio among women with CFS. One study actually found a tendency for lower progesterone levels during the luteal phase and higher follicle-stimulating hormone (FSH) levels in premenopausal women with CFS compared with controls. ${ }^{34}$ In agreement with the hypothesis of hormonal imbalance (lower progesterone levels) in CFS are the reports on worsening of CFS symptoms around menopause [CFS Bulletin] or improvement with the addition of estrogen/progesterone. ${ }^{33}$

Meaningful assessment of the relationship between CFS and female HR was not possible in our study because women were asked only about current HR but not when replacement started relative to menopause onset, what they took, or for how long. However, it is biologically plausible that low ovarian hormones may play some role in the pathogenesis of CFS in women because ovarian hormones can impact cognition, pain, mood, and numerous autonomic functions. ${ }^{35}$ Many of the symptoms typical for CFS, such as musculoskeletal pain, arthralgias, disturbed sleep, and cognitive impairment ("living in fog"), can be triggered by surgically (oophorectomy) or chemically induced menopause. We hypothesize that at any age, reduced or out of balance levels of gonadal hormones may contribute to the development of CFS symptoms through several known pathogenetic mechanisms: impaired sleep, ${ }^{36}$ loss of neuroprotective effects of progesterone and estradiol, ${ }^{37}$ increase in proinflammatory cytokines/upregulation of inflammation, ${ }^{38,39}$ possibly increased pain perception due to loss of estradiol's and progesterone's effects on antinociceptive pathways, ${ }^{40}$ and reduction of estrogen-facilitated and progesterone-facilitated healing and repair mechanisms. ${ }^{41,42}$

Various reasons can lead to gradual or sudden decline in production of female sex hormones. Examples include premature ovarian failure, hysterosalpingo-oophorectomy (not followed by HR), reduced production of gonadotropins at the hypothalamo-pituitary level (via stress or disease), and iatrogenically induced reversible hypogonadism (via use of gonadotropin-releasing hormone $[\mathrm{GnRH}]$ analogues). Interestingly, a case of FM (a condition that shares many symptoms with CFS) has been reported to develop directly after treatment with GnRH analogue. ${ }^{43}$

The hypothalamo-pituitary adrenal axis (and, especially, cortisol as a major stress hormone) has been relatively well studied in CFS. Early life stress and posttraumatic stress disorder (PTSD) have been found to be significant risk factors for
CFS. ${ }^{44,45}$ Given that stress unfavorably affects the production of sex steroids via the hypothalamo-pituitary gonadal axis, the potential link between stress and gonadal steroid hormones in CFS may need to be explored in future research.

Interpretation of our findings should be considered in view of the study's strengths and limitations. A major strength of the study is its design as a population-based case-control study, avoiding referral biases inherent to studies of CFS from referral clinics and the use of convenience controls. Nevertheless, the majority of the population in Wichita is white, and caution should be exercised in extrapolating conclusions to the entire U.S. population. However, our sample had basic gynecological health characteristics very similar to those from national data: mean age at menarche 12.4 vs. 12.7 years (from a study in three different states ${ }^{46}$ ), mean number of pregnancies/woman $2.0 \pm 0.2$ vs. fertility rate of 2.1/ woman, ${ }^{47}$ and mean age at natural menopause $49.2 \pm 1.6$ years in our controls vs. means of 49.0 to 50.5 years in national data. ${ }^{47,48}$

The study limitations include its relatively small sample size (with limited power to detect small effects as statistically significant), the limited number of questions (e.g., no questions on number of miscarriages and live births, on polycystic ovaries or infertility, and on age at hysterectomy), and selfreporting of conditions without review of medical records. The gynecological history was collected conveniently for examining its relationship to unexplained pelvic pain. No review of medical records was planned, as it was not feasible at this time and because exploring risk factors from the gynecological history was not a primary hypothesis in this study. Our findings are derived from a population-based study in which most patients with CFS reported gradual onset of symptoms and may not apply to women with sudden onset or postinfectious fatigue. Participants in this study were somewhat older than in other studies. ${ }^{8}$ A different participation rate between cases and controls may also have introduced selection bias. Nevertheless, our findings are consistent with the few studies examining relationships between CFS and gynecological history and with similar studies of FM.

In conclusion, the higher prevalence of gynecological conditions and surgeries in women with CFS highlights the importance of evaluating gynecological health in these patients. A thorough gynecological history should always be obtained in women with CFS or FM, and addressing gynecological problems and potential hormonal imbalances should be part of the overall care of women with CFS. The chronological (and pathophysiological) relationship among endometriosis, hysterectomy, and CFS remains to be examined in future, larger studies. Finally, it is obvious that not all women with the gynecological risk factors identified here develop CFS. Therefore, the interplay of potential genetic and environmental factors that affect gynecological function and health also needs to be examined in future CFS research. Better understanding of the various comorbidities and abnormalities associated with CFS (or subgrouping of the syndrome) could provide insights into its pathogenesis and, eventually, its prevention and treatment in affected women.

\section{Acknowledgments}

We acknowledge Elizabeth Unger, M.D., and Daisy Lee of the CDC and Suzanne Vernon, Ph.D., formerly of the CDC, 
for their contributions to the study protocol and Marjorie Morrissey and Rebecca Devlin of Abt Associates for managing the study. We thank all women who volunteered to participate in the study. The findings and conclusions in this report are those of the authors and do not necessarily represent those of the funding agency.

\section{Authors' Disclaimer}

The findings and conclusions in this report are those of the authors and do not necessarily represent the views of the Centers for Disease Control and Prevention.

\section{Disclosure Statement}

The authors have no conflicts of interest.

\section{References}

1. Gunn WJ, Connell DB, Randall B. Epidemiology of chronic fatigue syndrome: The Centers for Disease Control study. In: Bock BR, Whelan J, eds. Chronic fatigue syndrome. New York: John Wiley \& Sons, 1993:83-101.

2. Reyes M, Gary HE Jr, Dobbins JG, et al. Surveillance for chronic fatigue syndrome-Four U.S. cities, September 1989 through August 1993. MMWR CDC Surveill Summ. 1997; 46:1-13. [85\% were women]

3. Jason LA, Richman JA, Rademaker AW, et al. A communitybased study of chronic fatigue syndrome. Arch Intern Med 1999;159:2129-2137.

4. Reeves WC, Jones JF, Maloney E, et al. Prevalence of chronic fatigue syndrome in metropolitan, urban, and rural Georgia. Population Health Metrics 2007;5:5.

5. Fukuda K, Straus SE, Hickie I, Sharpe MC, Dobbins JG, Komaroff $\mathrm{A}$. The chronic fatigue syndrome: A comprehensive approach to its definition and study. International Chronic Fatigue Syndrome Study Group. Ann Intern Med 1994;121:953-959.

6. Reeves WC, Lloyd A, Vernon SD, et al. Identification of ambiguities in the 1994 chronic fatigue syndrome research case definition and recommendations for resolution. BMC Health Serv Res 2003;3:25.

7. Reeves WC, Wagner D, Nisenbaum R, et al. Chronic fatigue syndrome-A clinically empirical approach to its definition and study. BMC Med 2005;3:19.

8. Reyes M, Dobbins JG, Mawle AC, et al. Risk factors for chronic fatigue syndrome: A case-control study. J Chronic Fatigue Syndr 1996;2:17-33.

9. Harlow BL, Signorello LB, Hall JE, Dailey C, Komaroff AL. Reproductive correlates of chronic fatigue syndrome. Am J Med. 1998;105:94S-99S.

10. Reyes M, Nisenbaum R, Hoaglin DC, et al. Prevalence and incidence of chronic fatigue syndrome in Wichita, Kansas. Arch Intern Med 2003;163:1530-1536.

11. Ware JE Jr, Sherbourne CD. The MOS 36-item short-form health survey (SF-36). I. Conceptual framework and item selection. Med Care 1992;30:473-483.

12. Smets EM, Garssen B, Bonke B, De Haes JC. The Multidimensional Fatigue Inventory (MFI) psychometric qualities of an instrument to assess fatigue. J Psychosom Res 1995;39:315-325.

13. Wagner D, Nisenbaum R, Heim C, Jones JF, Unger ER, Reeves WC. Psychometric properties of the CDC Symptom Inventory for assessment of chronic fatigue syndrome. Popul Health Metr 2005;3:8.
14. Audebert A. Women with endometriosis: Are they different from others? Gynecol Obstet Fertil 2005;33:239-246.

15. Butrick CW. Patients with chronic pelvic pain: Endometriosis or interstitial cystitis/painful bladder syndrome? J Soc Laparoendoscop Surg. 2007;11:182-189.

16. Tietjen GE, Bushnell CD, Herial NA, Utley C, White L, Hafeez F. Endometriosis is associated with prevalence of comorbid conditions in migraine. Headache 2007;47:1069-1078.

17. Sinaii N, Cleary SD, Ballweg ML, Nieman LK, Stratton P. High rates of autoimmune and endocrine disorders, fibromyalgia, chronic fatigue syndrome and atopic diseases among women with endometriosis: A survey analysis. Hum Reprod 2002;17:2715-2724.

18. Hamilton WT, Gallagher AM, Thomas JM, White PD. Risk markers for both chronic fatigue syndrome and irritable bowel syndrome: A prospective case-control study in primary care. Psychol Med 2009;39:1913-1921.

19. Moorman PG, Schildkraut JM, Iversen ES, et al. A prospective study of weight gain after premenopausal hysterectomy. J Womens Health 2009;18:699-708.

20. Dickersin K, Lemaire GS. Hysterectomy. In: Goldman MB, Hatch M, eds. Women and health, San Diego: Academic Press, 2000:253-267.

21. chfs.ky.gov/NR/rdonlyres/8E019F58-6F80-4367-9E9A-C655 2F8B3B92/0/hadhyst.pdf Accessed September 2009.

22. www.scdhec.gov/hs/epidata/BRFSS/2008/hadhyst2.html Accessed September 2009.

23. Brett KM, Higgins JA. Hysterectomy prevalence by Hispanic ethnicity: Evidence from a national survey. Am J Public Health 2003;93:307-312.

24. Waxman J, Zatzkis SM. Fibromyalgia and menopause. Examination of the relationship. Postgrad Med 1986;80:165167, 170-171.

25. Wagener P, Hein R, Felstehausen KH. Gynecologic operations in fibromyalgia syndrome. A retrospective analysis of 890 patients of a rheumatologic and general practice. Fortschr Med 1997;115:39-40.

26. ter Borg EJ, Gerards-Rociu E, Haanen HC, Westers P. High frequency of hysterectomies and appendectomies in fibromyalgia compared with rheumatoid arthritis: A pilot study. Clin Rheumatol 1999;18:1-3.

27. Pamuk ON, Dönmez S, Cakir N. Increased frequencies of hysterectomy and early menopause in fibromyalgia patients: A comparative study. Clin Rheumatol 2009;28:561-564.

28. Rocca WA, Bower JH, Maraganore DM, et al. Increased risk of cognitive impairment or dementia in women who underwent oophorectomy before menopause. Neurology 2007; 69:1074-1083.

29. Rivera CM, Grossardt BR, Rhodes DJ, Rocca WA. Increased mortality for neurological and mental diseases following early bilateral oophorectomy. Neuroepidemiology 2009;33: 32-40.

30. Zhang Y, Lee ET, Cowan LD, North KE, Wild RA, Howard BV. Hysterectomy prevalence and cardiovascular disease risk factors in American Indian women. Maturitas 2005; 52:328-336.

31. Howard BV, Kuller L, Langer R, et al. Risk of cardiovascular disease by hysterectomy status, with and without oophorectomy: The Women's Health Initiative Observational Study. Circulation 2005;111:1462-1470.

32. Allison MA, Manson JE, Langer RD, et al. Oophorectomy, hormone therapy, and subclinical coronary artery disease in women with hysterectomy: The Women's Health Initiative Coronary Artery Calcium Study. Menopause 2008;15:639-647. 
33. Studd J, Panay N. Chronic fatigue syndrome. Lancet 1996; 348:1384.

34. Cevik R, Gur A, Acar S, Nas K, Sarac AJ. Hypothalamicpituitary-gonadal axis hormones and cortisol in both menstrual phases of women with chronic fatigue syndrome and effect of depressive mood on these hormones. BMC Musculoskelet Disord 2004;5:47.

35. Bethea CL, Pecins-Thompson M, Schutzer WE, Gundlah C, $\mathrm{Lu} \mathrm{ZN}$. Ovarian steroids and serotonin neural function. Mol Neurobiol 1998;18:87-123.

36. Eichling PS, Sahni J. Menopause related sleep disorders. J Clin Sleep Med 2005;1:291-300.

37. Roof RL, Hall ED. Gender differences in acute CNS trauma and stroke: Neuroprotective effects of estrogen and progesterone. J Neurotrauma 2000;17:367-388.

38. Pacifici R, Brown C, Puscheck E, et al. Effect of surgical menopause and estrogen replacement on cytokine release from human blood mononuclear cells. Proc Natl Acad Sci USA 1991;88:5134-5138.

39. Dijsselbloem N, Vanden Berghe W, De Naeyer A, Haegeman G. Soy isoflavone phyto-pharmaceuticals in interleukin-6 affections. Multi-purpose nutraceuticals at the crossroad of hormone replacement, anti-cancer and anti-inflammatory therapy. Biochem Pharmacol 2004;68:1171-1185.

40. Dawson-Basoa M, Gintzler AR. Gestational and ovarian sex steroid antinociception: Synergy between spinal kappa and delta opioid systems. Brain Res 1998;794:61-67.

41. Routley CE, Ashcroft GS. Effect of estrogen and progesterone on macrophage activation during wound healing. Wound Repair Regen 2009;17:42-50.
42. Engeland CG, Sabzehei B, Marucha PT. Sex hormones and mucosal wound healing. Brain Behav Immun. 2009;23: 629-635.

43. Toussirot E, Wendling D. Fibromyalgia developed after administration of gonadotrophin-releasing hormone analogue. Clin Rheumatol 2001;20:150-152.

44. Heim C, Wagner D, Maloney E, et al. Early adverse experience and risk for chronic fatigue syndrome: Results from a population-based study. Arch Gen Psychiatry 2006;63: 1258-1266.

45. Heim C, Nater UM, Maloney E, Boneva R, Jones JF, Reeves WC. Childhood trauma and risk for chronic fatigue syndrome: Association with neuroendocrine dysfunction. Arch Gen Psychiatry 2009;66:72-80.

46. Nichols HB, Trentham-Dietz A, Hampton JM, et al. From menarche to menopause: Trends among U.S. women born from 1912 to 1969. Am J Epidemiology 2006;164:1003-1011.

47. National Vital Statistics Reports, 2009; 57:33.

48. Mondul AM, Rodriguez C, Jacobs EJ, Calle EE. Age at natural menopause and cause-specific mortality. Am J Epidemiol 2005;162:1089-1097.

Address correspondence to: Roumiana S. Boneva, M.D., Ph.D. Centers for Disease Control and Prevention Mailstop A-15 1600 Clifton Road, NE Atlanta, GA 30333

E-mail: rboneva@cdc.gov 